Bulletin of the Section of Logic

Volume 45/2 (2016), pp. 111-124

http://dx.doi.org/10.18778/0138-0680.45.2.04

George Voutsadakis

\title{
CATEGORICAL ABSTRACT LOGIC: HIDDEN MULTI-SORTED LOGICS AS MULTI-TERM $\pi$-INSTITUTIONS
}

\begin{abstract}
Babenyshev and Martins proved that two hidden multi-sorted deductive systems are deductively equivalent if and only if there exists an isomorphism between their corresponding lattices of theories that commutes with substitutions. We show that the $\pi$-institutions corresponding to the hidden multi-sorted deductive systems studied by Babenyshev and Martins satisfy the multi-term condition of Gil-Férez. This provides a proof of the result of Babenyshev and Martins by appealing to the general result of Gil-Férez pertaining to arbitrary multi-term $\pi$-institutions. The approach places hidden multi-sorted deductive systems in a more general framework and bypasses the laborious reuse of well-known proof techniques from traditional abstract algebraic logic by using "off the shelf" tools.

Keywords: Behavioral Equivalence, Hidden Logic, Multi-Sorted Logic, Multi-term $\pi$-Institutions, Interpretability, Deductive Equivalence.
\end{abstract}

\section{Introduction}

In [13], the author introduced a framework in which the deductive equivalence of two logical systems formalized as $\pi$-institutions may be studied. In Lemma 9.4 of [13] it was proved that if two $\pi$-institutions $\mathcal{I}$ and $\mathcal{I}^{\prime}$ are deductively equivalent, then there exists an adjoint equivalence between their categories of theories that commutes with substitutions. The converse of this result does not hold for arbitrary $\pi$-institutions (see Section 9 of [9]). The notion of a term $\pi$-institution was also introduced in [13] and 
it was proved that for term $\pi$-institutions the converse holds, i.e., if there exists an adjoint equivalence between the categories of theories of two term $\pi$-institutions that commutes with substitutions, then the two $\pi$-institutions are deductively equivalent (see Theorem 10.5 of [13]).

This equivalence result was extended in [9], where a more general notion than that of a term $\pi$-institution, that of a multi-term $\pi$-institution, was introduced and it was shown that two multi-term $\pi$-institutions are deductively equivalent if and only if there exists an adjoint equivalence between their categories of theories that commutes with substitutions (see Theorem 8.9 of [9]).

These results were further enhanced in subsequent work, first, by Galatos and Tsinakis [8] and, more recently, by Galatos and Gil-Férez [7], that partly based their investigations on preceding work by Blok and Jónsson [3]. However, these more recent expansions of the theory will not concern us in this paper, since the level of generality of [9] is adequate for our purposes.

In [10], Martins initiated the study of behavioral reasoning in hidden logics in the context of abstract algebraic logic (see, also, [11]). Behavioral algebraization of logics was also expounded upon in [5] in which the logical systems are multi-sorted, but there is only a single visible sort of formulas, which are the formulas in the contexts of which experiments can be performed with visible or observable outputs/outcomes. The work of [5] was extended to a categorical level in [14].

In [1], the scope of the framework of [5] was extended to cover the case of hidden multi-sorted logical systems (HMsLs) with multiple visible sorts and their behavioral algebraization was introduced. The main theorem, Theorem 4.5, of [1] is more widely encompassing than preceding results in the framework of behavioral logics. One of the main drawbacks of its proof, however, is that its technical details are almost identical to those employed in establishing previously obtained results of a similar kind in abstract algebraic logic, e.g., Theorem 3.7 of [4].

We undertake in this work the task of showing how the powerful methods of categorical abstract algebraic logic may be employed, within a rather limited scope and with rather moderate force, as compared to their full power, to establish results of this kind, avoiding a tedious reapplication of a long and cumbersome process from scratch.

In fact the present paper, apart from its technical details may be viewed as a manifesto in advertising the appropriateness and suitability of employ- 
ing abstract equivalence results for logical systems from categorical abstract algebraic logic with the purpose of, not only avoiding repetition of identical or similar proofs for similar equivalence results, but, also, placing them in a hierarchy of "equivalence strengths", which may be roughly described by the equivalences studied in $[3,13,9,8,7]$ and $[6]$.

\section{Behavioral Equivalence of HMsLs}

We borrow the following treatment of hidden multi-sorted logical systems from the work of Babenyshev and Martins [1]. We omit details pertaining to the basics of multi-sorted set theory which are intuitively clear, since many details may be found in [1] and more, if necessary, in [10, 12].

A hidden (sorted) signature is a triple $\Sigma=\left\langle\mathrm{SORT}, \mathrm{VIS},\left\langle\mathrm{OP}_{\tau}: \tau \epsilon\right.\right.$ TYPE $\rangle$, where

- SORT is a nonempty countable set of sorts;

- $\mathrm{VIS} \subseteq \mathrm{SORT}$ is the set of visible sorts;

- TYPE is a set of nonempty sequences $S_{0}, \ldots, S_{n-1}, S_{n}$ of sorts, called types, written $S_{0}, \ldots, S_{n-1} \rightarrow S_{n}$, and, for all $\tau \in \mathrm{TYPE}, \mathrm{OP}_{\tau}$ is a countable set of operation symbols of type $\tau$. We denote $\mathrm{OP}=$ $\left\langle\mathrm{OP}_{\tau}: \tau \in \mathrm{TYPE}\right\rangle$.

Sorts in HID $=$ SORT $\backslash$ VIS are called hidden sorts. Elements of $\mathrm{OP}_{\tau}$ are operation symbols of type $\tau$ and those of type $\rightarrow S$ are called constants. We assume that all sets of operation symbols are disjoint. For a hidden signature $\Sigma$, the associated unhidden signature $\Sigma^{\mathrm{UH}}$ is the one obtained from $\Sigma$ by making all sorts visible. We call two hidden signatures $\Sigma, \Sigma^{\prime}$ algebraically indistinguishable if $\Sigma^{\mathrm{UH}}=\Sigma^{\prime \mathrm{UH}}$.

Let $X=\left\langle X_{S}: S \in \mathrm{SORT}\right\rangle$ be a fixed countably infinite sorted set of (sorted propositional) variables with pairwise disjoint components. Terms are formed in the usual way starting from the variables in $X$ and using the operation symbols in $\mathrm{OP}$ respecting types. We denote by $\mathrm{Te}_{\Sigma}(X)$ the SORT-sorted set of terms over $\Sigma$ with variables in $X$. Since the components of $\mathrm{Te}_{\Sigma}(\mathrm{X})$ are pairwise disjoint, we may identify any (sorted) subset $\Gamma \subseteq \mathrm{Te}_{\Sigma}(\mathrm{X})$ with the union $\bigcup_{S \in \mathrm{SORT}} \Gamma_{S}$.

If the sorted signature $\Sigma$ has a ground term of every sort (i.e., one without variables), it will be called standard.

Let $k \geq 1$ be a fixed natural number. A $k$-variable of sort $S$ is a sequence of $k$-variables all of sort $S$. A $k$-term or $k$-formula of sort $S$ is 
a sequence of $k$-terms of sort $S$. We write $\bar{\varphi}: S=\left\langle\varphi_{0}(\bar{x}): S, \ldots, \varphi_{k-1}(\bar{x}): S\right\rangle$ for such a $k$-term of sort $S$ and denote the set of $k$-terms of sort $S$ by $\left(\mathrm{Te}_{\Sigma}(\mathrm{X})\right)_{\mathrm{S}}^{\mathrm{k}}$. Set $\mathrm{Te}_{\Sigma}^{\mathrm{k}}(\mathrm{X})=\left\langle\left(\mathrm{Te}_{\Sigma}(\mathrm{X})\right)_{\mathrm{S}}^{\mathrm{k}}: \mathrm{S} \in \mathrm{SORT}\right\rangle$. The set of visible $k$-terms is $\left(\mathrm{Te}_{\Sigma}^{\mathrm{k}}(\mathrm{X})\right)_{\mathrm{VIS}}=\left\langle\left(\mathrm{Te}_{\Sigma}(\mathrm{X})\right)_{\mathrm{V}}^{\mathrm{k}}: \mathrm{V} \in \mathrm{VIS}\right\rangle$.

A sorted function or mapping $h$ from a SORT-sorted set $A=\left\langle A_{S}: S \epsilon\right.$ $\mathrm{SORT}\rangle$ to a SORT-sorted set $B=\left\langle B_{S}: S \in \mathrm{SORT}\right\rangle$, in symbols $h: A \rightarrow B$, is a sorted set $h=\left\langle h_{S}: S \in \mathrm{SORT}\right\rangle$ of functions such that $h_{S}: A_{S} \rightarrow B_{S}$, for each $S \in \mathrm{SORT}$. We define, in the usual way, operations over $\operatorname{Te}_{\Sigma}(X)$ to obtain the term algebra $\mathbf{T e}_{\Sigma}(X)$ over the signature $\Sigma$. Then $\mathbf{T e}_{\Sigma}(X)$ has the universal mapping property over $X$ in the sense that, for every $\Sigma$-algebra $\boldsymbol{A}$ and every sorted map $h: X \rightarrow A$, called an assignment, there is a unique sorted homomorphism $h^{*}: \mathbf{T e}_{\Sigma}(X) \rightarrow \boldsymbol{A}$ that extends $h$. As usual no notational distinction is made between $h$ and $h^{*}$. A map from $X$ to the set of terms, and its unique extension to an endomorphism of $\operatorname{Te}_{\Sigma}(X)$, is called a substitution.

A hidden $k$-logic is a pair $\mathcal{L}=\langle\Sigma, \vdash \mathcal{L}\rangle$, where $\Sigma$ is a hidden signature with VIS the set of visible sorts and $\vdash \mathcal{L} \subseteq \mathcal{P}\left(\left(\mathrm{Te}_{\Sigma}^{\mathrm{k}}(\mathrm{X})\right)_{\mathrm{VIS}}\right) \times\left(\mathrm{Te}_{\Sigma}^{\mathrm{k}}(\mathrm{X})\right)_{\mathrm{VIS}}$ an unsorted relation satisfying, for all $\Gamma \cup \Delta \cup\{\bar{\gamma}, \bar{\varphi}\} \subseteq\left(\mathrm{Te}_{\Sigma}^{\mathrm{k}}(\mathrm{X})\right)_{\mathrm{VIS}}$,

(i) $\Gamma r_{\mathcal{L}} \bar{\gamma}$, for all $\bar{\gamma} \in \Gamma$;

(ii) $\Gamma r_{\mathcal{L}} \bar{\varphi}$ and $\Delta \vdash_{\mathcal{L}} \bar{\gamma}$, for all $\bar{\gamma} \in \Gamma$, imply $\Delta \vdash_{\mathcal{L}} \bar{\varphi}$;

(iii) $\Gamma \vdash_{\mathcal{L}} \bar{\varphi}$ implies $\sigma(\Gamma) \vdash_{\mathcal{L}} \sigma(\bar{\varphi})$, for every (sorted) substitution $\sigma$ : $\mathrm{Te}_{\Sigma}(\mathrm{X}) \rightarrow \mathrm{Te}_{\Sigma}(\mathrm{X})$.

A SORT-sorted set $A=\left\langle A_{S}: S \in \mathrm{SORT}\right\rangle$ is called locally finite if, for every $S \in \mathrm{SORT}, A_{S}$ is a finite set. Moreover $A$ is said to be globally finite if $A$ is locally finite and $A_{S}$ is empty except for a finite number of sorts.

Let $\mathcal{L}=\left\langle\Sigma, \vdash_{\mathcal{L}}\right\rangle$ be a hidden $k$-logic. The relation $\vdash_{\mathcal{L}}$ is finitary if $\Gamma \vdash_{\mathcal{L}} \bar{\varphi}$ implies $\Delta \vdash \bar{\varphi}$, for some globally finite subset $\Delta$ of $\Gamma$. We say that $\mathcal{L}$ is specifiable if $r_{\mathcal{L}}$ is finitary. We say $\mathcal{L}$ is standard if $\Sigma$ is standard. We say $\mathcal{L}$ is a visible $k$-logic if VIS $=$ SORT.

A set of visible $k$-terms $T$ closed under $\vdash_{\mathcal{L}}$, i.e., such that $T r_{\mathcal{L}} \bar{\varphi}$ implies $\bar{\varphi} \in T$, is called a theory of $\mathcal{L}$ or an $\mathcal{L}$-theory. The set of all $\mathcal{L}$-theories is denoted by $\operatorname{Th}(\mathcal{L})$. It is well known that, under set-theoretic inclusion, this set forms a complete lattice $\operatorname{Th}(\mathcal{L})$. This lattice is algebraic if the logic $\mathcal{L}$ is specifiable.

Let $\Sigma, \Sigma^{\prime}$ be two algebraically indistinguishable signatures with sets of visible sorts VIS and $\mathrm{VIS}^{\prime}$, respectively. A $(k, \ell)$-translation from $\Sigma$ 
to $\Sigma^{\prime}$ is a VIS-VIS'-sorted set $\tau=\left\langle\tau_{R}(\bar{x}: R): R \in\right.$ VIS $\rangle$, where $\tau_{R}(\bar{x}:$ $R)=\left\langle\bar{\tau}_{R, V}(\bar{x}: R): V \in \mathrm{VIS}^{\prime}\right\rangle$ is a $\mathrm{VIS}^{\prime}$-sorted set of visible terms, with $\bar{\tau}_{R, V}(\bar{x}: R)$ a finite set of $\ell$-terms over $\Sigma^{\prime}$ of sort $V$, with variables in $\bar{x}=\left\langle x_{0}: R, \ldots, x_{k-1}: R\right\rangle$.

A translation $\tau$ is an interpretation from the hidden $k$-logic $\mathcal{L}$ to the hidden $\ell$-logic $\mathcal{L}^{\prime}$ over hidden signatures $\Sigma$ and $\Sigma^{\prime}$, respectively, if, for all $\Gamma \cup\{\bar{\varphi}\} \subseteq\left(\mathrm{Te}_{\Sigma}^{\mathrm{k}}(\mathrm{X})\right)_{\mathrm{VIS}}$,

$$
\Gamma \vdash_{\mathcal{L}} \bar{\varphi} \quad \text { iff } \quad \tau(\Gamma) \vdash_{\mathcal{L}^{\prime}} \tau(\bar{\varphi}) .
$$

Given two algebraically indistinguishable hidden signatures $\Sigma, \Sigma^{\prime}$, a hidden $k$-logic $\mathcal{L}$ over $\Sigma$ and a hidden $\ell$-logic $\mathcal{L}^{\prime}$ over $\Sigma^{\prime}, \mathcal{L}$ is equivalent to $\mathcal{L}^{\prime}$ if there are globally finite interpretations (i.e., with only finitely many nonempty components, each of finite cardinality) $\tau$ and $\rho$ from $\mathcal{L}$ to $\mathcal{L}^{\prime}$ and from $\mathcal{L}^{\prime}$ to $\mathcal{L}$, respectively, such that

$$
\bar{x}: V \dashv \vdash_{\mathcal{L}} \rho(\tau(\bar{x}: V)) \quad \text { and } \quad \bar{y}: V^{\prime} \dashv \vdash_{\mathcal{L}^{\prime}} \tau\left(\rho\left(\bar{y}: V^{\prime}\right)\right),
$$

for all $V \in$ VIS and all $V^{\prime} \in$ VIS $^{\prime}$.

Let $\Sigma, \Sigma^{\prime}$ be two algebraically indistinguishable hidden signatures and $\mathcal{L}, \mathcal{L}^{\prime}$ a hidden $k$-logic and a hidden $\ell$-logic over $\Sigma, \Sigma^{\prime}$, respectively. A map $\alpha: \operatorname{Th}(\mathcal{L}) \rightarrow \operatorname{Th}\left(\mathcal{L}^{\prime}\right)$ commutes with substitutions if, for every substitution $\sigma$ and all $T \in \operatorname{Th}(\mathcal{L})$,

$$
C_{\mathcal{L}^{\prime}}(\sigma(\alpha(T)))=\alpha\left(C_{\mathcal{L}}(\sigma(T))\right),
$$

where $C_{\mathcal{L}}$ and $C_{\mathcal{L}^{\prime}}$ denote the closure operators associated with the consequence operators $\vdash_{\mathcal{L}}$ and $\vdash_{\mathcal{L}^{\prime}}$, respectively.

The main result of Babenyshev and Martins, Theorem 4.5 of [1], is the following characterization of the equivalence of two hidden multi-sorted logical systems in terms of the existence of a complete lattice isomorphism between their corresponding lattices of theories that commutes with substitutions:

Theorem 1 (Babenyshev and Martins [1]). Let $\mathcal{L}$ and $\mathcal{L}^{\prime}$ be a $k$ - and an $\ell$-hidden standard, specifiable logic over algebraically indistinguishable hidden signatures $\Sigma$ and $\Sigma^{\prime}$, respectively. Then $\mathcal{L}$ and $\mathcal{L}^{\prime}$ are equivalent iff there exists an isomorphism from $\mathbf{T h}(\mathcal{L})$ to $\mathbf{T h}\left(\mathcal{L}^{\prime}\right)$ that commutes with substitutions. 


\section{Cofibrations and Grothendieck Construction}

To pass to a brief account of the treatment of the equivalence of multi-term $\pi$-institutions of [9], we first need a few categorical preliminaries pertaining to cofibrations. We borrow these from [2], even though a few details are also offered in [9], as the theory is developed.

Let $P: \mathbf{E} \rightarrow \mathbf{C}$ be a functor between small categories, $f \in \mathbf{C}(C, D)$ and $P(X)=C$. Then an arrow $u \in \mathbf{E}(X, Y)$ is cocartesian for $f$ and $X$ if

- $P(u)=f$ and

- for any $v \in \mathbf{E}(X, Z)$ and $k \in \mathbf{C}(D, P(Z))$, such that $k \circ f=P(v)$, there exists unique $w \in \mathbf{E}(Y, Z)$, such that $w \circ u=v$ and $P(w)=k$.
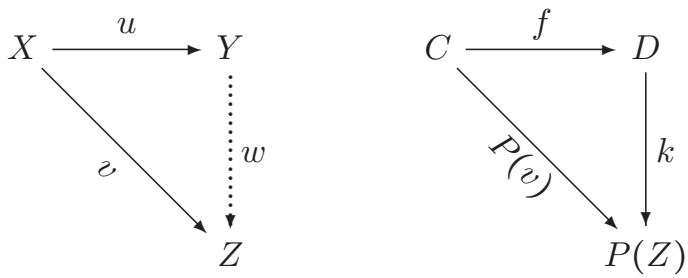

The functor $P: \mathbf{E} \rightarrow \mathbf{C}$ is a cofibration if there exists a cocartesian arrow for all $f \in \mathbf{C}(C, D)$ and all $X \in|\mathbf{E}|$, such that $P(X)=C$.

A cocleavage for a cofibration $P: \mathbf{E} \rightarrow \mathbf{C}$ is a function $\kappa$ that takes $f \in \mathbf{C}(C, D)$ and $X \in|\mathbf{E}|$, such that $P(X)=C$, to an arrow $\kappa(f, X)$ that is cocartesian for $f$ and $X$.

The cocleavage $\kappa$ is called a splitting of a cofibration $P: \mathbf{E} \rightarrow \mathbf{C}$ if

- $\kappa\left(i_{C}, X\right)=i_{X}$, for all $P(X)=C$, and

- $\kappa(g, Y) \circ \kappa(f, X)=\kappa(g \circ f, X)$, for all $f \in \mathbf{C}(C, D)$ and $g \in \mathbf{C}(D, E)$, $P(X)=C$ and $Y$ the codomain of $\kappa(f, X)$.

A split cofibration is one that possesses a splitting.

If $P: \mathbf{E} \rightarrow \mathbf{C}$ and $P^{\prime}: \mathbf{E}^{\prime} \rightarrow \mathbf{C}^{\prime}$ are two cofibrations over $\mathbf{C}$ and $\mathbf{C}^{\prime}$, respectively, a morphism of cofibrations from $P$ to $P^{\prime}$ is a pair of functors $\langle\zeta, \xi\rangle$, such that the following diagram commutes:

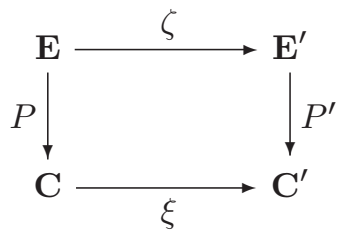


If $P, P^{\prime}$ are split cofibrations with splittings $\kappa$ and $\kappa^{\prime}$, respectively, a morphism of cofibrations $\langle\zeta, \xi\rangle: P \rightarrow P^{\prime}$ is a morphism of split cofibrations (relative to $\kappa$ and $\kappa^{\prime}$ ) if and only if, for all $X \in|\mathbf{E}|$ and $f \in \mathbf{C}(P(X), D)$,

$$
\zeta(\kappa(f, X))=\kappa^{\prime}(\xi(f), \zeta(X)) .
$$

A functor $F: \mathbf{E} \rightarrow \mathbf{C}$ is said to be exhaustive on objects if for every $C \in|\mathbf{C}|$, there exists $X \in|\mathbf{E}|$, such that $F(X)=C$. It is exhaustive on arrows if, for every $f$ in $\mathbf{C}$, there exists $u$ in $\mathbf{E}$, such that $F(u)=f$.

In Proposition 5.3 of [9], it is shown that, given two cofibrations $P: \mathbf{E} \rightarrow \mathbf{C}, P^{\prime}: \mathbf{E}^{\prime} \rightarrow \mathbf{C}^{\prime}$, with $P$ exhaustive on objects (and, hence, also on arrows) and $\langle\zeta, \xi\rangle: P \rightarrow P^{\prime}$ a morphism of cofibrations, then $\xi$ is determined by $\zeta$, since $\xi$ is the unique functor $\chi: \mathbf{C} \rightarrow \mathbf{C}^{\prime}$, such that $\langle\zeta, \chi\rangle: P \rightarrow P^{\prime}$ is a morphism of cofibrations. Thus, in this setting, one is justified using the notation $\xi=\zeta^{+}$for $\xi$ and writing $\left\langle\zeta, \zeta^{+}\right\rangle: P \rightarrow P^{\prime}$.

Consider, next a functor $F: \mathbf{C} \rightarrow$ Cat. Define the Grothendieck construction (of $F$ over $\mathbf{C}$ ) to be the category $\int_{\mathbf{C}} F=\mathbf{G}(\mathbf{C}, F)$ specified as follows:

GC1 An object of $\mathbf{G}(\mathbf{C}, F)$ is a pair $(x, C)$, where $C \in|\mathbf{C}|$ and $x$ is an arrow in $F(C)$;

GC2 An arrow $(u, f):(x, C) \rightarrow\left(x^{\prime}, C^{\prime}\right)$ has $f: C \rightarrow C^{\prime}$ an arrow of $\mathbf{C}$ and $u: F(f)(x) \rightarrow x^{\prime}$ an arrow of $F\left(C^{\prime}\right)$;

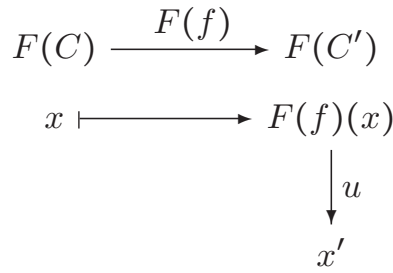

GC3 If $(u, f):(x, C) \rightarrow\left(x^{\prime}, C^{\prime}\right)$ and $(v, g):\left(x^{\prime}, C^{\prime}\right) \rightarrow\left(x^{\prime \prime}, C^{\prime \prime}\right)$,

$$
F(f)(x) \stackrel{u}{\longrightarrow} x^{\prime} \quad F(g)\left(x^{\prime}\right) \stackrel{v}{\longrightarrow} x^{\prime \prime}
$$

then $(v, g) \circ(u, f):(x, C) \rightarrow\left(x^{\prime \prime}, C^{\prime \prime}\right)$ is defined by

$$
\begin{gathered}
F(g)(F(f)(x)) \stackrel{F(g)(u)}{\longrightarrow} F(g)\left(x^{\prime}\right) \longrightarrow x^{\prime \prime} \\
(v, g) \circ(u, f)=(v \circ F(g)(u), g \circ f) .
\end{gathered}
$$

It turns out that the following result holds (Theorem 12.2.9 of [2]): 
Theorem 2. Given a functor $F: \mathbf{C} \rightarrow \mathbf{C a t}, \mathbf{G}(\mathbf{C}, F)$ is a category and the second projection is a functor $P: \mathbf{G}(\mathbf{C}, F) \rightarrow \mathbf{C}$ which is a split cofibration with splitting

$$
\kappa(f,(x, C))=\left(i_{F(f)(x)}, f\right):(x, C) \rightarrow\left(F(f)(x), C^{\prime}\right)
$$

for any arrow $f: C \rightarrow C^{\prime}$ of $\mathbf{C}$ and object $(x, C)$ of $\mathbf{G}(\mathbf{C}, F)$.

\section{Equivalence of Multi-Term $\pi$-Institutions}

A $\pi$-institution is a triple $\mathcal{I}=\langle\operatorname{Sign}, \mathrm{SEN}, C\rangle$, where $\mathbf{S i g n}$ is a category of signatures, SEN : Sign $\rightarrow$ Set is a sentence functor and $C=\left\{C_{\Sigma}\right\}_{\Sigma \in|\operatorname{Sign}|}$ is a closure system on SEN, i.e., a $\mid$ Sign|-indexed family of closure operators $C_{\Sigma}: \mathcal{P}(\operatorname{SEN}(\Sigma)) \rightarrow \mathcal{P}(\operatorname{SEN}(\Sigma))$ that satisfy

$$
\operatorname{SEN}(f)\left(C_{\Sigma}(\Phi)\right) \subseteq C_{\Sigma^{\prime}}(\operatorname{SEN}(f)(\Phi)),
$$

for all $\Sigma, \Sigma^{\prime} \in|\operatorname{Sign}|$, all $f \in \operatorname{Sign}\left(\Sigma, \Sigma^{\prime}\right)$ and all $\Phi \subseteq \operatorname{SEN}(\Sigma)$.

Given a $\pi$-institution $\mathcal{I}$, the category of sentences $\operatorname{Sen}(\mathcal{I})$ is the Grothendieck construction $\operatorname{Sen}(\mathcal{I})=\int_{\text {Sign }}$ SEN. Modulo some obvious identifications, we may view its objects as pairs $\langle\Sigma, \varphi\rangle$, where $\Sigma \in|\mathbf{S i g n}|$ and $\varphi \in \operatorname{SEN}(\Sigma)$. Its arrows $f:\langle\Sigma, \varphi\rangle \rightarrow\left\langle\Sigma^{\prime}, \varphi^{\prime}\right\rangle$ are Sign-morphisms $f: \Sigma \rightarrow \Sigma^{\prime}$, such that $\operatorname{SEN}(f)(\varphi)=\varphi^{\prime}$. The objects of this category are called $\mathcal{I}$-sentences.

If $\mathcal{I}=\langle\operatorname{Sign}, \operatorname{SEN}, C\rangle$ is a $\pi$-institution, an endofunctor $\Upsilon: \operatorname{Sen}(\mathcal{I}) \rightarrow$ $\operatorname{Sen}(\mathcal{I})$ is called a multi-source signature-variable (mssv) (see Definition 3.6 of [9]) if, for every pair $\langle\Sigma, \varphi\rangle,\left\langle\Sigma^{\prime}, \varphi^{\prime}\right\rangle \in|\operatorname{Sen}(\mathcal{I})|$, if $g:\langle\Sigma, \varphi\rangle \rightarrow$ $\left\langle\Sigma^{\prime}, \varphi^{\prime}\right\rangle$ is in $\operatorname{Sen}(\mathcal{I})$, then $\Upsilon(\langle\Sigma, \varphi\rangle)=\Upsilon\left(\left\langle\Sigma^{\prime}, \varphi^{\prime}\right\rangle\right)$ and $\Upsilon(g)=1_{\Upsilon(\langle\Sigma, \varphi\rangle)}$ and, there exists a natural transformation $\kappa: \Upsilon \rightarrow I_{\operatorname{Sen}(\mathcal{I})}$. Thus, for every $g:\langle\Sigma, \varphi\rangle \rightarrow\left\langle\Sigma^{\prime}, \varphi^{\prime}\right\rangle$ in $\operatorname{Sen}(\mathcal{I})$, the following diagram commutes:

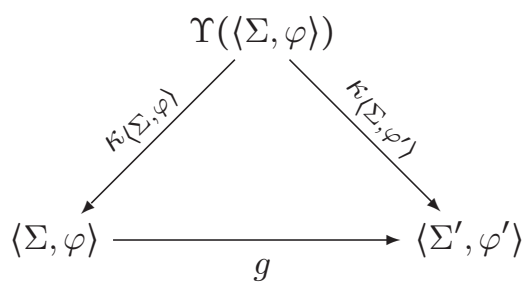

A $\pi$-institution $\mathcal{I}$ is called multi-term ([9], Definition 3.8) (or is said to satisfy the multi-term condition) if it has a multi-source signaturevariable. 
Given a $\pi$-institution $\mathcal{I}=\langle\operatorname{Sign}, \mathrm{SEN}, C\rangle$, the theory functor of $\mathcal{I}$ is the functor Th : Sign $\rightarrow$ CoLat (the category of complete lattices), such that, for all $\Sigma \in|\operatorname{Sign}|, \operatorname{Th}(\Sigma)=\mathcal{C}_{\Sigma}$ (the closed set system corresponding to the closure operator $C_{\Sigma}$ ) and, for every $f \in \operatorname{Sign}\left(\Sigma, \Sigma^{\prime}\right), \operatorname{Th}(f): \mathcal{C}_{\Sigma} \rightarrow \mathcal{C}_{\Sigma^{\prime}}$ is the lattice morphism given by

$$
\operatorname{Th}(f)(T)=C_{\Sigma^{\prime}}(\operatorname{SEN}(f)(T)), \text { for all } T \in \mathcal{C}_{\Sigma} .
$$

The category of theories $\operatorname{Th}(\mathcal{I})$ of $\mathcal{I}$ is the Grothendieck construction $\operatorname{Th}(\mathcal{I})=\int_{\text {Sign }}$ Th of Th over Sign. More explicitly, again modulo some obvious identifications, the objects are pairs $\langle\Sigma, T\rangle$, with $\Sigma \epsilon|\operatorname{Sign}|$ and $T \epsilon$ $|\operatorname{Th}(\Sigma)|$. Given $\langle\Sigma, T\rangle,\left\langle\Sigma^{\prime}, T^{\prime}\right\rangle \in|\mathbf{T h}(\mathcal{I})|$, a morphism $f:\langle\Sigma, T\rangle \rightarrow\left\langle\Sigma^{\prime}, T^{\prime}\right\rangle$ is an arrow $f \in \operatorname{Sign}\left(\Sigma, \Sigma^{\prime}\right)$, such that $\operatorname{SEN}(f)(T) \subseteq T^{\prime}$.

We denote by SIG : $\mathbf{T h}(\mathcal{I}) \rightarrow$ Sign the second projection, which, by Theorem 2, is a split cofibration.

Let SEN : Sign $\rightarrow$ Set and $\mathrm{SEN}^{\prime}: \mathbf{S i g n}^{\prime} \rightarrow$ Set be two sentence functors. A translation $\langle F, \alpha\rangle: \mathrm{SEN} \rightarrow \mathrm{SEN}^{\prime}$ from $\mathrm{SEN}$ to $\mathrm{SEN}^{\prime}$ consists of a functor $F$ : Sign $\rightarrow$ Sign' $^{\prime}$ together with a natural transformation $\alpha: \mathrm{SEN} \rightarrow \mathcal{P} \operatorname{SEN}^{\prime} F$. Let $\mathcal{I}=\langle\mathbf{S i g n}, \mathrm{SEN}, C\rangle, \mathcal{I}^{\prime}=\left\langle\mathbf{S i g n}^{\prime}, \mathrm{SEN}^{\prime}, C^{\prime}\right\rangle$ be $\pi$-institutions. A semi-interpretation of $\mathcal{I}$ in $\mathcal{I}^{\prime}$ is a translation $\langle F, \alpha\rangle$ : $\mathrm{SEN} \rightarrow \mathrm{SEN}^{\prime}$, such that, for every $\Sigma \in|\operatorname{Sign}|$ and all $\Gamma \cup\{\varphi\} \subseteq \operatorname{SEN}(\Sigma)$,

$$
\varphi \in C_{\Sigma}(\Gamma) \text { implies } \alpha_{\Sigma}(\varphi) \subseteq C_{F(\Sigma)}^{\prime}\left(\alpha_{\Sigma}(\Gamma)\right) .
$$

An interpretation of $\mathcal{I}$ in $\mathcal{I}^{\prime}$ is a translation $\langle F, \alpha\rangle: \mathrm{SEN} \rightarrow \mathrm{SEN}^{\prime}$, such that

$$
\varphi \in C_{\Sigma}(\Gamma) \text { if and only if } \alpha_{\Sigma}(\varphi) \subseteq C_{F(\Sigma)}^{\prime}\left(\alpha_{\Sigma}(\Gamma)\right),
$$

for every $\Sigma \in|\operatorname{Sign}|$ and all $\Gamma \cup\{\varphi\} \subseteq \operatorname{SEN}(\Sigma)$. We write $\langle F, \alpha\rangle: \mathcal{I}\rangle \mathcal{I}^{\prime}$ for semi-interpretations and $\langle F, \alpha\rangle: \mathcal{I} \vdash \mathcal{I}^{\prime}$ for interpretations.

Let $\mathcal{I}, \mathcal{I}^{\prime}$ be $\pi$-institutions and $\langle F, \alpha\rangle: \mathrm{SEN} \rightarrow \mathrm{SEN}^{\prime}$ a translation. Define the functor $F^{\alpha}: \operatorname{Th}(\mathcal{I}) \rightarrow \operatorname{Th}\left(\mathcal{I}^{\prime}\right)$ as follows:

1. $F^{\alpha}(\langle\Sigma, T\rangle)=\left\langle F(\Sigma), C_{F(\Sigma)}^{\prime}\left(\alpha_{\Sigma}(T)\right)\right\rangle$, for all $\langle\Sigma, T\rangle \in|\mathbf{T h}(\mathcal{I})|$;

2. $F^{\alpha}(f)=F(f)$, for all $f:\langle\Sigma, T\rangle \rightarrow\left\langle\Sigma^{\prime}, T^{\prime}\right\rangle$ in $\mathbf{T h}(\mathcal{I})$. If $\operatorname{SEN}(f)(T) \subseteq T^{\prime}$, then $\operatorname{SEN}(F(f))\left(C_{F(\Sigma)}^{\prime}\left(\alpha_{\Sigma}(T)\right)\right) \subseteq C_{F\left(\Sigma^{\prime}\right)}^{\prime}\left(\alpha_{\Sigma^{\prime}}\left(T^{\prime}\right)\right)$, and the definition makes sense.

Note that, with this definition of $F^{\alpha}$, the pair $\left\langle F^{\alpha}, F\right\rangle: \mathrm{SIG} \rightarrow \mathrm{SIG}^{\prime}$ becomes a morphism of cofibrations. 


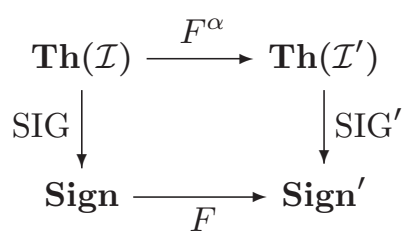

Let $\mathcal{I}=\langle$ Sign, $\mathrm{SEN}, C\rangle, \mathcal{I}^{\prime}=\left\langle\mathbf{S i g n}^{\prime}, \mathrm{SEN}^{\prime}, C^{\prime}\right\rangle$ be two $\pi$-institutions. $\mathcal{I}$ is deductively equivalent to $\mathcal{I}^{\prime}$ if and only if there exist interpretations $\langle F, \alpha\rangle: \mathcal{I} \vdash \mathcal{I}^{\prime},\langle G, \beta\rangle: \mathcal{I}^{\prime} \vdash \mathcal{I}$ and an adjoint equivalence $\langle F, G, \eta, \varepsilon\rangle$ : Sign $\rightarrow$ Sign $^{\prime}$, such that

(i) for all $\Sigma \in|\operatorname{Sign}|, \operatorname{Th}\left(\eta_{\Sigma}\right) \mathrm{C}_{\Sigma}=\left(\mathrm{G}^{\beta} \mathrm{F}^{\alpha}\right)_{\Sigma} \mathrm{C}_{\Sigma}$;

(ii) for all $\Sigma^{\prime} \in\left|\mathbf{S i g n}^{\prime}\right|, \operatorname{Th}\left(\varepsilon_{\Sigma^{\prime}}\right)\left(\mathrm{F}^{\alpha} \mathrm{G}^{\beta}\right)_{\Sigma^{\prime}} \mathrm{C}_{\Sigma^{\prime}}^{\prime}=\mathrm{C}_{\Sigma^{\prime}}^{\prime}$.

Returning to categories of theories, given two $\pi$-institutions $\mathcal{I}, \mathcal{I}^{\prime}$, as above, a functor $F: \operatorname{Th}(\mathcal{I}) \rightarrow \operatorname{Th}\left(\mathcal{I}^{\prime}\right)$ is signature respecting in the sense of [13] if and only if $\left\langle F, F^{+}\right\rangle: \mathrm{SIG} \rightarrow \mathrm{SIG}^{\prime}$ is a morphism of cofibrations for a unique functor $F^{+}$: Sign $\rightarrow$ Sign' $^{\prime}$ (see Remark 5.4 of [9]):

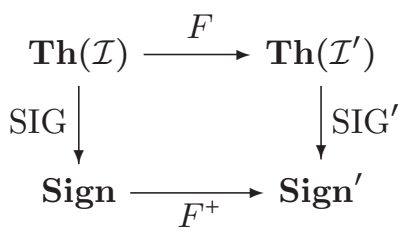

If, given $\langle\Sigma, T\rangle \in|\mathbf{T h}(\mathcal{I})|$, we denote the second component of $F(\langle\Sigma, T\rangle)$ by $F_{\Sigma}(T)$, we have, under the hypothesis of signature respectability,

$$
F(\langle\Sigma, T\rangle)=\left\langle F^{+}(\Sigma), F_{\Sigma}(T)\right\rangle .
$$

Let $\mathcal{I}, \mathcal{I}^{\prime}$ be two $\pi$-institutions and $F: \operatorname{Th}(\mathcal{I}) \rightarrow \operatorname{Th}\left(\mathcal{I}^{\prime}\right)$ be signature respecting, which forces $\left\langle F, F^{+}\right\rangle$to be a morphism of cofibrations. Then, Theorem 5.6 of [9] shows that $\left\langle F, F^{+}\right\rangle$is a morphism of split cofibrations if and only if, for all $\Sigma, \Sigma^{\prime} \in|\operatorname{Sign}|$, all $f \in \operatorname{Sign}\left(\Sigma, \Sigma^{\prime}\right)$ and all $\langle\Sigma, T\rangle \in$ $|\operatorname{Th}(\mathcal{I})|$,

$$
F_{\Sigma^{\prime}}\left(C_{\Sigma^{\prime}}(\operatorname{SEN}(f)(T))\right)=C_{F^{+}\left(\Sigma^{\prime}\right)}^{\prime}\left(\operatorname{SEN}^{\prime}\left(F^{+}(f)\right)\left(F_{\Sigma}(T)\right)\right),
$$

i.e., if and only if $F: \operatorname{Th}(\mathcal{I}) \rightarrow \operatorname{Th}\left(\mathcal{I}^{\prime}\right)$ commutes with substitutions in the sense of [13].

Gil-Férez established the following main theorem, Theorem 8.9 of [9], generalizing Theorem 10.5 of [13]: 
Theorem 3. If $\mathcal{I}=\langle\mathbf{S i g n}, \mathrm{SEN}, C\rangle$ and $\mathcal{I}^{\prime}=\left\langle\mathbf{S i g n}^{\prime}, \mathrm{SEN}^{\prime}, C^{\prime}\right\rangle$ are two multi-term $\pi$-institutions, then $\mathcal{I}$ and $\mathcal{I}^{\prime}$ are deductively equivalent iff there exists an adjoint equivalence $\langle F, G, \eta, \varepsilon\rangle: \mathbf{T h}(\mathcal{I}) \rightarrow \mathbf{T h}\left(\mathcal{I}^{\prime}\right)$ that commutes with substitutions.

\section{HMsLs as Multi-Term $\pi$-Institutions}

Let $\Sigma$ be a hidden signature and consider a hidden $k$-logic $\mathcal{L}=\left\langle\Sigma, \vdash_{\mathcal{L}}\right\rangle$ over $\Sigma$. The $\pi$-institution $\mathcal{I}_{\mathcal{L}}$ associated with $\mathcal{L}$ is $\mathcal{I}_{\mathcal{L}}=\left\langle\mathrm{M}_{\Sigma}\right.$, SEN, $\left.C_{\mathcal{L}}\right\rangle$, where:

- $\mathbf{M}_{\Sigma}$ is the category determined by the monoid of all SORT-sorted substitutions $\sigma: \mathrm{Te}_{\Sigma}(\mathrm{X}) \rightarrow \mathrm{Te}_{\Sigma}(\mathrm{X})$, with the single object $\star$.

- SEN : $\mathbf{M}_{\Sigma} \rightarrow$ Set is the functor, such that $\mathrm{SEN}(\star)=\left(\mathrm{Te}_{\Sigma}^{\mathrm{k}}(\mathrm{X})\right)_{\mathrm{VIS}}$ and, for all $\sigma$ in $\mathbf{M}_{\Sigma}, \operatorname{SEN}(\sigma):\left(\operatorname{Te}_{\Sigma}^{\mathrm{k}}(\mathrm{X})\right)_{\mathrm{VIS}} \rightarrow\left(\mathrm{Te}_{\Sigma}^{\mathrm{k}}(\mathrm{X})\right)_{\mathrm{VIS}}$ maps $\bar{\varphi}: V$ to $\sigma(\bar{\varphi}): V$.

- $C_{\mathcal{L}}=\left\{C_{\mathcal{L}}\right\}$ is the closure operator on $\left(\mathrm{Te}_{\Sigma}^{\mathrm{k}}(\mathrm{X})\right)_{\mathrm{VIS}}$ determined by the consequence operator $\vdash_{\mathcal{L}}$, i.e., such that $C_{\mathcal{L}}(\Gamma)=\left\{\bar{\varphi} \in\left(\mathrm{Te}_{\Sigma}^{\mathrm{k}}(\mathrm{X})\right)_{\mathrm{VIS}}\right.$ : $\left.\Gamma \vdash_{\mathcal{L}} \bar{\varphi}\right\}$, for all $\Gamma \subseteq\left(\mathrm{Te}_{\Sigma}^{\mathrm{k}}(\mathrm{X})\right)_{\mathrm{VIS}}$.

We now show that, given a $k$-hidden logic $\mathcal{L}=\left\langle\Sigma, \vdash_{\mathcal{L}}\right\rangle$ that is standard and specifiable, the $\pi$-institution $\mathcal{I}_{\mathcal{L}}=\left\langle\mathbf{M}_{\Sigma}, \mathrm{SEN}, C_{\mathcal{L}}\right\rangle$ is multi-term.

Suppose $\mathcal{L}=\left\langle\Sigma, \vdash_{\mathcal{L}}\right\rangle$ is a standard specifiable hidden $k$-logic over a hidden signature $\Sigma=\left\langle\mathrm{SORT}\right.$, VIS, $\left.\left\langle\mathrm{OP}_{\tau}: \tau \in \mathrm{TYPE}\right\rangle\right\rangle$. Let $X=\left\langle X_{S}: S \in\right.$ SORT $\rangle$ with $X_{S}=\left\{x_{1}: S, x_{2}: S, \ldots\right\}$ be an enumeration of the set $X_{S}$ of $S$-sorted propositional variables, where the $X_{S}$ 's are assumed pairwise disjoint.

For every $S \in \mathrm{SORT}$, let $\bar{x}: S=\left\langle x_{1}: S, x_{2}: S, \ldots, x_{k}: S\right\rangle$. Let $\Upsilon$ : $\operatorname{Sen}\left(\mathcal{I}_{\mathcal{L}}\right) \rightarrow \operatorname{Sen}\left(\mathcal{I}_{\mathcal{L}}\right)$ be the functor defined by:

- $\Upsilon(\langle\star, \bar{\varphi}: V\rangle)=\langle\star, \bar{x}: V\rangle$, for all $\langle\star, \bar{\varphi}: V\rangle \in\left|\operatorname{Sen}\left(\mathcal{I}_{\mathcal{L}}\right)\right|$;

- for all $g:\langle\star, \bar{\varphi}: V\rangle \rightarrow\left\langle\star, \bar{\psi}: V^{\prime}\right\rangle$ in $\operatorname{Sen}\left(\mathcal{I}_{\mathcal{L}}\right), \Upsilon(g)=i_{\mathrm{Te}_{\Sigma}(\mathrm{X})}$.

Since $g:\langle\star, \bar{\varphi}: V\rangle \rightarrow\left\langle\star, \bar{\psi}: V^{\prime}\right\rangle$ is in $\operatorname{Sen}\left(\mathcal{I}_{\mathcal{L}}\right)$ only if $V=V^{\prime}$, it suffices to show that there exists a natural transformation from $\Upsilon$ to $\mathrm{I}_{\operatorname{Sen}\left(\mathcal{I}_{\mathcal{L}}\right)}$ :

For $\bar{\varphi}: V \in\left(\mathrm{Te}_{\Sigma}^{\mathrm{k}}(\mathrm{X})\right)_{\mathrm{VIS}}$, let $\kappa^{\bar{\varphi}: V}: \mathrm{Te}_{\Sigma}(\mathrm{X}) \rightarrow \mathrm{Te}_{\Sigma}(\mathrm{X})$ be the SORTsorted endomorphism on $\mathrm{Te}_{\Sigma}(\mathrm{X})$, such that

(i) For $S=V, i \leq k, \kappa^{\bar{\varphi}: V}\left(x_{i}: V\right)=\varphi_{i}$;

(ii) For $S=V, i>k, \kappa^{\bar{\varphi}: V}\left(x_{i}: V\right)=\varphi_{k}$; 
(iii) For $S \neq V$ and all $x \in X_{S}, \kappa^{\bar{\varphi}: V}(x: S)=t: S$, where $t: S$ is a fixed ground term of sort $S$, which exists since $\mathcal{L}$ is standard.

For all $\bar{\varphi}: V \in\left(\operatorname{Te}_{\Sigma}(\mathrm{X})\right)_{\mathrm{VIS}}, \operatorname{SEN}\left(\kappa^{\bar{\varphi}: V}\right)(\bar{x}: V)=\bar{\varphi}: V$. So by setting $\kappa_{\langle\star, \bar{\varphi}: V\rangle}=\kappa^{\bar{\varphi}: V}$, we get that $\kappa: \Upsilon \rightarrow \mathrm{I}_{\operatorname{Sen}\left(\mathcal{I}_{\mathcal{L}}\right)}$ is a transformation and it now suffices to show that it is natural.

To see this, given $g:\langle\star, \bar{\varphi}: V\rangle \rightarrow\langle\star, \bar{\psi}: V\rangle$, we get:

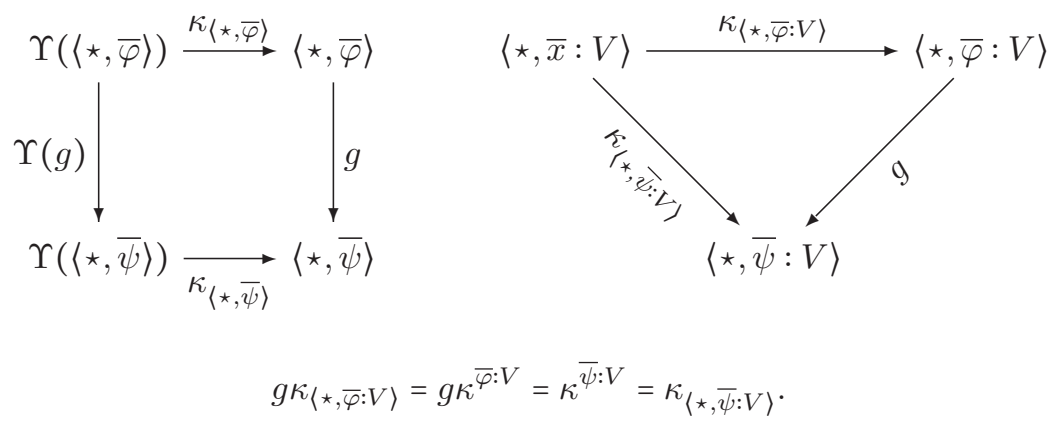

So $\kappa$ is indeed a natural transformation, $\Upsilon$ is a multi-source signaturevariable and $\mathcal{I}_{\mathcal{L}}$ is a multi-term $\pi$-institution.

THEOREM 4. Let $\mathcal{L}$ and $\mathcal{L}^{\prime}$ be a standard, specifiable $k$ - and a standard specifiable $\ell$-hidden logic over algebraically indistinguishable hidden signatures $\Sigma$ and $\Sigma^{\prime}$, respectively. Then $\mathcal{L}$ and $\mathcal{L}^{\prime}$ are equivalent iff there exists an isomorphism from $\operatorname{Th}(\mathcal{L})$ to $\operatorname{Th}\left(\mathcal{L}^{\prime}\right)$ that commutes with substitutions.

Proof: $\mathcal{L}$ and $\mathcal{L}^{\prime}$ are equivalent iff $\mathcal{I}_{\mathcal{L}}$ and $\mathcal{I}_{\mathcal{L}^{\prime}}$ are deductively equivalent iff, by Theorem 3 , there exists an adjoint equivalence $\langle F, G, \eta, \varepsilon\rangle: \mathbf{T h}\left(\mathcal{I}_{\mathcal{L}}\right) \rightarrow$ $\operatorname{Th}\left(\mathcal{I}_{\mathcal{L}^{\prime}}\right)$ that commutes with substitutions iff, since the categories of signatures are monoids, there exists an isomorphism from $\operatorname{Th}(\mathcal{L})$ to $\operatorname{Th}\left(\mathcal{L}^{\prime}\right)$ that commutes with substitutions.

We showed in Theorem 4 that Theorem 4.5 of [1] (Theorem 1) can be obtained as a corollary of Theorem 8.9 of [9] (Theorem 3). We advocate that the machinery developed by the work on equivalence be employed, when possible, instead of reusing tedious and heavily technical proofs from scratch. Besides avoiding repetition, this application of "off the shelf" tools would serve well in exploring how the various pieces fit in the general (meta-meta-)theory and, also, in revealing interconnections between them that may remain obscure or, perhaps, even hidden, otherwise. 


\section{Acknowledgements}

This work owes its existence to preceding work of Gil-Férez on the equivalence of multi-term $\pi$-institutions and on work of Martins and his collaborators on the behavioral equivalence of various types of hidden multisorted deductive systems, i.e., systems over multi-sorted signatures, some of whose sorts may be declared hidden (inaccessible to the "user"). The author gratefully acknowledges the scientific debt to both works.

Thanks go also to an anonymous referee who noticed a number of oversights and, thus, contributed in improving the presentation.

\section{References}

[1] S. Babenyshev and M. A. Martins, Behavioral Equivalence of Hidden k-Logics: An Abstract Algebraic Approach, Journal of Applied Logic, Vol. 16 (2016), pp. 72-91.

[2] M. Barr and C. Wells, Category Theory for Computing Science, Third Edition, Les Publications CRM, Montréal, 1999.

[3] W. J. Blok and B. Jónsson, Equivalence of Consequence Operations, Studia Logica, Vol. 83, No. 1/3 (2006), pp. 91-110.

[4] W. J. Blok and D. Pigozzi, Algebraizable Logics, Memoirs of the American Mathematical Society, Vol. 77, No. 396 (1989).

[5] C. Caleiro, R. Gon calves and M. Martins, Behavioral Algebraization of Logics, Studia Logica, Vol. 91, No. 1 (2009), pp. 63-111.

[6] J. M. Font and T. Moraschini, M-Sets and the Representation Problem, Studia Logica, Vol. 103, No. 1 (2015), pp. 21-51.

[7] Galatos, N., and N. Galatos, J. Gil-Férez, Modules over Quantaloids: Applications to the Isomorphism Problem in Algebraic Logic and $\pi$-institutions, Journal of Pure and Applied Algebra, Vol. 221, No. 1 (2017), pp. 1-24.

[8] N. Galatos and C. Tsinakis, Equivalence of Closure Operators: an OrderTheoretic and Categorical Perspective, The Journal of Symbolic Logic, Vol. 74, No. 3 (2009), pp. 780-810.

[9] J. Gil-Férez, Multi-term $\pi$-Institutions and their Equivalence, Mathematical Logic Quarterly, Vol. 52, No. 5 (2006), pp. 505-526.

[10] M. A. Martins, Behavioral Reasoning in Generalized Hidden Logics, Ph.D. Thesis, Faculdade de Ciências, University of Lisbon, 2004. 
[11] M. A. Martins and D. Pigozzi, Behavioural Reasoning for Conditional Equations, Mathematical Structures in Computer Science, Vol. 17 (2007), pp. 1075-1113.

[12] D. Sannella and A. Tarlecki, Foundations of Algebraic Specification and Formal Software Development, EATCS Monographs in Theoretical Computer Science, Springer 2012.

[13] G. Voutsadakis, Categorical Abstract Algebraic Logic: Equivalent Institutions, Studia Logica, Vol. 74 (2003), pp. 275-311.

[14] G. Voutsadakis, Categorical Abstract Algebraic Logic: Behavioral $\pi$-Institutions, Studia Logica, Vol. 102, No. 3 (2014), pp. 617-646.

School of Mathematics and Computer Science

Lake Superior State University

Sault Sainte Marie

MI 49783

USA

e-mail: gvoutsad@lssu.edu 